\title{
Leiomioma vascular bucal: relato de dois casos, revisão da literatura e estudo imuno-histoquímico
}

\section{Oral vascular leiomyoma: report of two cases, review of literature and immunohistochemistry study}

Vanessa Ávila Sarmento Silveira'; Susana Ungaro Amadei'; Ana Cristina Posch Machado1; Renata Falchete do Prado'; Adriana Costa Neves²; Maria Rozeli de Souza Quirino ${ }^{3}$; Yasmin Rodarte Carvalho ${ }^{4}$

\begin{tabular}{l|l}
\multicolumn{1}{c|}{ Unitermos } & resumo \\
Leiomioma & $\begin{array}{l}\text { Introdução: Os leiomiomas são neoplasias benignas do músculo liso que ocorrem mais comumente nos } \\
\text { tratos geniturinário e gastrintestinal, entretanto são raros na cavidade bucal, na qual provavelmente a maioria } \\
\text { Angiomioma }\end{array}$ \\
$\begin{array}{l}\text { Imuno-histoquimica } \\
\text { Aspectos histopatológicos }\end{array}$ & $\begin{array}{l}\text { casos de mulheres tem sua origem a partir de músculo liso vascular. Objetivos: Relatar dois casos com história } \\
\text { Após exame microscópico que revelou numerosos vasos sangüíneos entremeados a células fusiformes, com } \\
\text { núcleos ovalados ou alongados, e exame imuno-histoquímico com positividade para actina de músculo } \\
\text { liso nos dois casos, o diagnóstico final foi leiomioma vascular. Conclusão: O estudo do leiomioma vascular } \\
\text { bucal é de extrema importância devido à raridade e semelhança desse com outras lesões da cavidade bucal. } \\
\text { A análise imuno-histoquímica é importante para o diagnóstico final do leiomioma. }\end{array}$
\end{tabular}

abstract

Introduction: The leiomyomas are benign tumors of smooth muscle origin, which are more frequently found in genitourinary and gastrointestinal tracts. However, leiomyomas rarely arise in the oral cavity, and when it happens, the majority of them is originated by smooth muscle of blood vessels. Aims: Report two cases of oral vascular leiomyoma with similar clinical characteristics, and discuss their aspects with the literature. Case report: Two cases of vascular leiomyoma in women that presented a nodular mass in the tongue ventral surface were described. The microscopic analysis revealed numerous blood vessels surrounded by spindle cells with ovoid or elongated nuclei. The two cases presented immunopositivity for smooth muscle actin, confirming the diagnosis. Conclusion: The study of vascular leiomyoma is required due to its rarity and similar aspects with other oral common lesions. The immunohistochemical analysis is a fundamental tool for the final diagnosis of leiomyoma. key words Leiomyoma Angiomyoma Immunohistochemistry Histopathological aspects

\section{Introdução}

Os leiomiomas são neoplasias benignas originadas da proliferação das células da musculatura lisa, as quais podem ocorrer em qualquer região do corpo em que o tecido é encontrado, incluindo as paredes de vasos sangüíneos e linfáticos maiores ${ }^{(5,9,13,19)}$. De acordo com a classificação da World Health Organization (WHO), os leiomiomas podem ser divididos principalmente em angiomiomas e/ou vasculares, e em epitelióides, também chamados de leiomioblastomas ${ }^{(42)}$.

O útero é o sítio de localização mais comum, com incidência de acometimento de $95 \%$ dos $\operatorname{casos}^{(17)}$. As lesões também podem ser encontradas no trato gas-

\footnotetext{
1. Doutorandas em Biopatologia Bucal da Faculdade de Odontologia de São José dos Campos da Universidade Estadual Paulista (FOSJC/UNESP).

2. Doutora em Patologia Bucal da Faculdade de Odontologia da Universidade de São Paulo (USP).

3. Professora Doutora da Disciplina de Patologia Bucal e Diagnóstico Bucal do Departamento de Odontologia da Universidade de Taubaté (UNITAU).
}

4. Professora-adjunta da Disciplina de Patologia Bucal do Departamento de Biociências e Diagnóstico Bucal da FOSJC/UNESP. 
trintestinal, pele e tecido subcutâneo(10), mas raramente são observadas na cavidade bucal ${ }^{(17,38)}$.

A patogênese das neoplasias do músculo liso, exceto aquelas do trato genital feminino, é incerta ${ }^{(17)}$. A presença da musculatura lisa nos tecidos bucais que poderia dar origem a essas lesões tem sido investigada $(1,12,20,25,40)$. Os autores sugerem, principalmente, a túnica média da parede de vasos e outros sítios sugestivos, como as papilas circunvaladas ${ }^{(32)}$.

De acordo com a literatura, em 1884 foi descrito o primeiro caso desse tipo de neoplasia na boca e, até 1996, há registros na literatura de 139 casos de leiomiomas na cavidade bucal(43).

A maioria dos leiomiomas vasculares intrabucais é assintomática ${ }^{(1,6,7,23,43)}$. Poucos casos relatados na literatura apresentaram queixa de dor associada à lesão $0^{(24,41,43)}$. Essas lesões bucais podem ocorrer em qualquer idade $e^{(6,43)}$, sendo mais freqüentes em adultos de média idade ${ }^{(6,25)}$ do sexo masculino ${ }^{(2,6)}$. Segundo alguns autores, o sítio mais acometido é a língua, além de palato, mucosa da bochecha e lábio inferior ${ }^{(8,14,43)}$. A localização intra-óssea, apesar de rara, também foi relatada ${ }^{(4,26)}$.

Clinicamente o leiomioma bucal é caracterizado por um nódulo pequeno, bem delimitado, superficial e de crescimento lento ${ }^{(21)}$, apresentando geralmente 0,5 a 3 $\mathrm{cm}$ de tamanho( ${ }^{(16)}$, mas raramente é maior que $5 \mathrm{~cm}^{(43)}$. A coloração da lesão é variada, dependendo de sua vascularização e de sua localização em profundidade(21).

Ao exame microscópico, os angiomiomas consistem em nódulos bem delimitados, contendo numerosos vasos de paredes espessadas e com lúmen parcialmente evidente. Tipicamente, as camadas internas de músculo liso dos vasos são arranjadas em circunferência, enquanto as camadas externas se dispõem em espiral. Esses vasos são de difícil classificação, pois não se assemelham a artérias ou a veias típicas. Feixes entrelaçados de células fusiformes, com núcleos alongados de coloração pálida e extremidades rombas, também são observados ${ }^{(6,34)}$. Áreas com alterações mixóides, hialinizações e coleções de gordura podem ser encontradas. Figuras de mitoses são $\operatorname{raras}^{(34)}$. Em alguns casos, observam-se fibras nervosas, cuja presença pode estar associada a relato de sintomatologia dolorosa ${ }^{(19)}$.

Devido a algumas de suas características histopatológicas, o leiomioma vascular possui muitas similaridades com neurilemoma ${ }^{(9,41)}$ e neurofibroma, entre outros ${ }^{(2,9)}$. Para o diagnóstico pode ser necessária a análise histoquímica, utilizando-se, por exemplo, a coloração tricrômica de Masson ${ }^{(29,32)}$. A análise imu- no-histoquímica tornou-se uma importante ferramenta para o diagnóstico dessas lesões. Por se tratar de lesões freqüentemente circunscritas, com recorrência rara ${ }^{(6,44)}$, o tratamento recomendado é a excisão cirúrgica local ${ }^{(2,6,23,44)}$.

O estudo de lesões de ocorrência rara e o relato de novos casos são importantes, pois permitem reunir dados que levam ao conhecimento do comportamento biológico e prognóstico das mesmas.

Dessa forma, o objetivo deste trabalho é relatar dois casos de leiomioma vascular, expondo e discutindo suas características histológicas e imuno-histoquímicas.

\section{Relato dos casos}

\section{Caso 1}

Paciente M.N., 66 anos, leucoderma, sexo feminino, compareceu, em 2006, à Faculdade de Odontologia de São José dos Campos da Universidade Estadual Paulista (FOSJC/UNESP), apresentando nódulo submucoso de aproximadamente $2 \mathrm{~cm}$ de diâmetro, localizado no ventre lingual do lado direito, consistente à palpação e de coloração semelhante à mucosa. Com a hipótese clínica de neoplasia glandular, realizou-se biópsia excisional e o material foi enviado para exame histopatológico.

Microscopicamente, os cortes histológicos revelaram fragmentos de língua apresentando camada muscular esquelética, em cuja profundidade notava-se neoplasia mesenquimal parcialmente delimitada. As células neoplásicas exibiam núcleos ovalados, cromatina frouxa e distribuíamse aleatoriamente, entremeadas por abundante matriz intercelular. Em algumas regiões os núcleos apresentavam aspecto alongado, por vezes com ponta romba, e as células fusiformes se dispunham paralelamente, formando feixes. Além disso, permeando toda lesão, foram observados numerosos vasos sangüíneos de calibres variados (Figura 1). Na periferia da lesão também se observavam dissociação e degeneração de feixes de fibras musculares.

\section{Caso 2}

Paciente E.C.S., 50 anos, leucoderma, sexo feminino, compareceu, em 2002, à Faculdade de Odontologia da Universidade de Taubaté (FO/UNITAU), apresentando nódulo submucoso na região de ventre lingual (lado esquerdo) de consistência firme, com aproximadamente $1,5 \mathrm{~cm}$ no maior eixo, somente percebido à palpação. A mucosa que recobria a lesão apresentava-se íntegra. Com a hipótese 


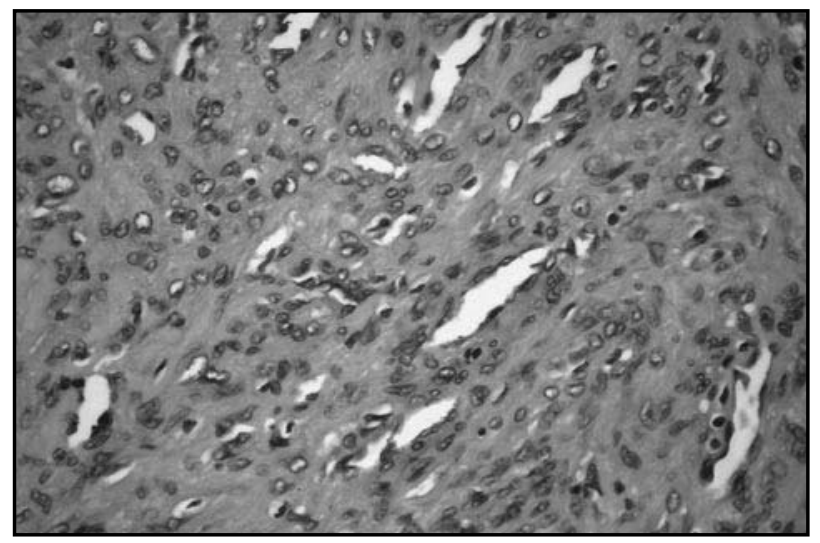

Figura 1 - Numerosos vasos sangüíneos de paredes espessadas em meio à proliferação celular (HE - aumento original 400 vezes)

clínica de adenoma pleomórfico, neuroma e/ou leiomioma, foi realizada biópsia excisional e o material enviado para exame histopatológico.

Microscopicamente, os cortes histológicos revelaram neoplasia benigna, bem delimitada e parcialmente encapsulada. Essas células estavam arranjadas em feixes que se orientavam em várias direções. A periferia da lesão apresentava-se bastante celularizada e também com grande número de vasos sangüíneos, diversos dos quais se mostravam com paredes espessadas, congestos e lúmen evidente (Figura 2).

No centro da lesão foram observados numerosos vasos sangüíneos entremeados a células fusiformes com núcleos ovalados ou alongados, por vezes com suas extremidades rombas. Notavam-se ainda regiões basofílicas e presença de alguns vacúolos no interstício. Próximo à neoplasia foram observados feixes de fibras musculares esqueléticas, glândulas salivares menores do tipo mucoso, com atrofia acinar e fibras nervosas. A coloração tricrômica de Masson revelou células neoplásicas coradas em vermelho-pálido.

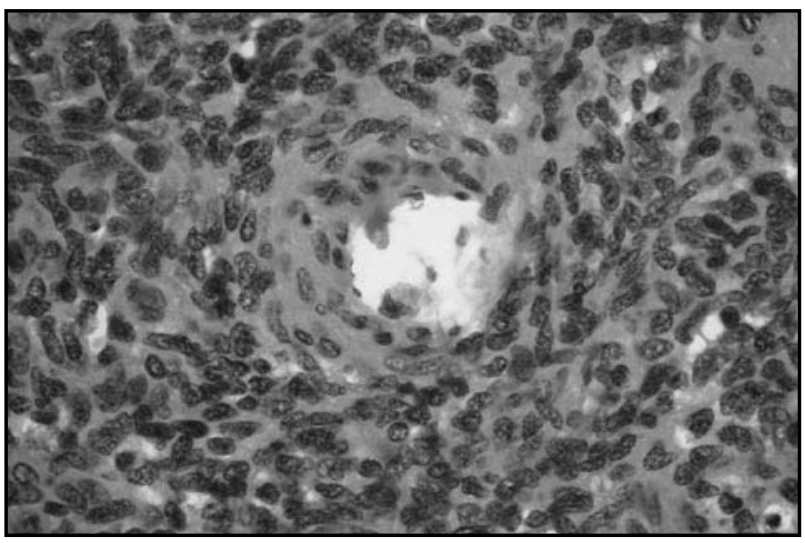

Figura 2 - Vaso sangüíneo de parede espessada com camadas celulares concêntricas e lúmen evidente ( $\mathrm{HE}$ - aumento original 400 vezes)

\section{Análise imuno-histoquímica}

Com as hipóteses de leiomioma vascular, neurofibroma e tumor fibroso solitário, as neoplasias foram submetidas à análise imuno-histoquímica.

A imuno-histoquímica foi realizada por meio da técnica da estreptavidina-biotina-peroxidase, utilizando-se anticorpos comercialmente disponíveis, com emprego de controles negativos e positivos apropriados. A origem, clone, concentração e período de incubação dos anticorpos primários monoclonais estão listados na Tabela 1.

O painel imuno-histoquímico foi composto basicamente pelos anticorpos contra actina de músculo liso (Figura 3), CD-34 (Figura 4) e proteína S-100. Vimentina (Figura 5), desmina e actina (HHF-35), CD-31 e citoqueratinas AE1/AE3 também foram utilizados.

Com base nos achados histopatológicos, associados a evidente imunoexpressão da actina de músculo liso, foram descartadas as hipóteses de neurofibroma e tumor fibroso solitário, sendo o diagnóstico final de leiomioma vascular bucal.

\section{Tabela 1 Anticorpos empregados, origem, concentração e período de incubação}

\begin{tabular}{lccc}
\hline Tipo & Clone & Diluição & Incubação (minutos) \\
Citoqueratina & Policlonal AE1/AE3 & $1: 75$ & 30 \\
CD-34 & QBend & $1: 50$ & 40 \\
CD-31 & PCAM1 & $1: 100$ & Overnight \\
S-100 & Policlonal & $1: 700$ & 30 \\
Vimentina & V 9 & $1: 800$ & 90 \\
AML & Clone 1A4 & $1: 300$ & 60 \\
Desmina & D33 & $1: 150$ & 60 \\
HHF-35 & HHF35(1,2) & $1: 50$ & 60 \\
\hline
\end{tabular}

AML: actina de músculo liso.

Fonte: Dako Corporation, Glosstrup, Denmark. 


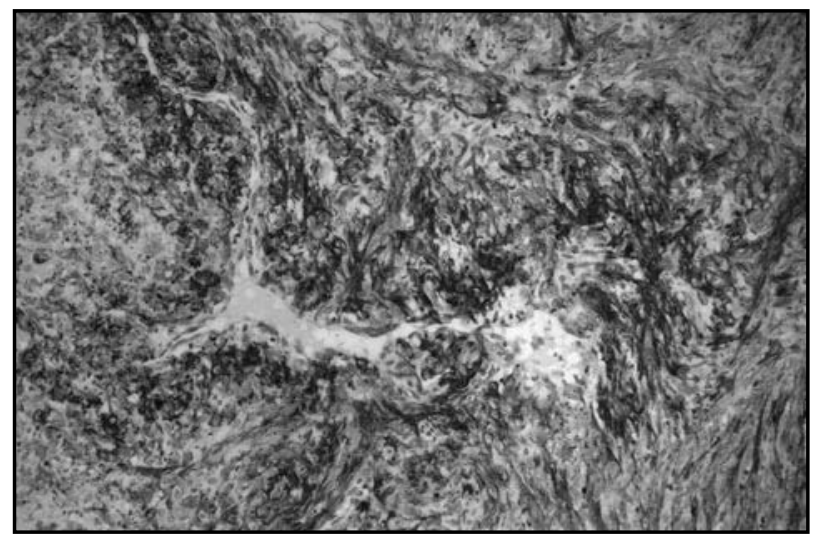

Figura 3 - Expressão da actina de músculo liso pelas células musculares neoplásicas. Ausência de marcação nas células endoteliais. (imuno-histoquímica, estreptavidinabiotina-peroxidase - aumento original 200 vezes)

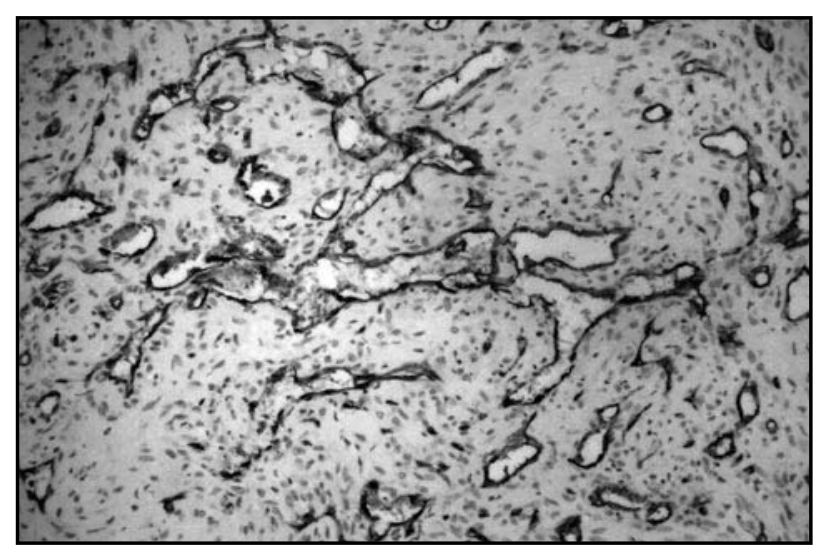

Figura 4 - Expressão do CD-34 pelas células endoteliais. Ausência de marcação nas células neoplásicas (imuno-histoquímica, estreptavidina-biotina-peroxidase - aumento original 200 vezes)

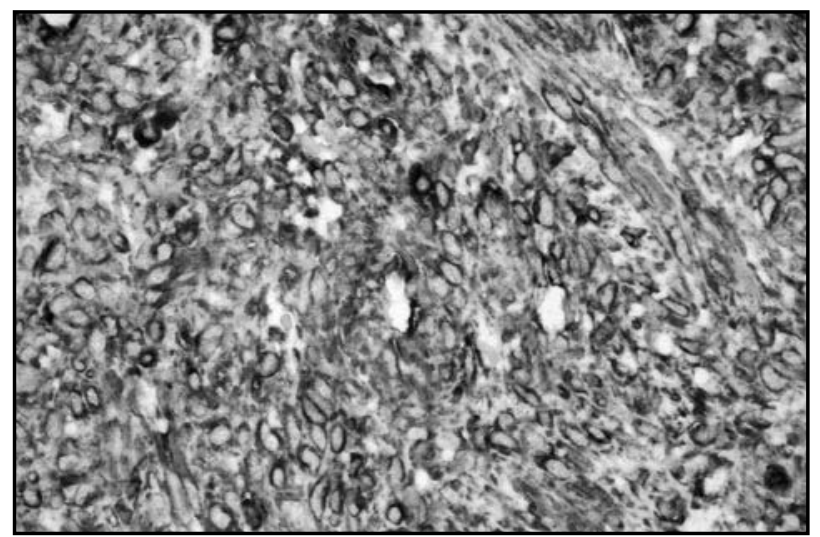

Figura 5 - Reação imuno-histoquímica positiva para vimentina (imuno-histoquímica, estreptavidina-biotina-peroxidase - aumento original 400 vezes)

\section{Discussão}

Os leiomiomas vasculares são neoplasias benignas originadas de músculo liso e pouco freqüentes na cavidade bucal(38), devido à presença pouco expressiva da musculatura lisa nessa região ${ }^{(9,11,27,37)}$. Sua etiologia permanece desconhecida ${ }^{(17)}$.
Os leiomiomas uterinos parecem ser estrógeno-dependentes ${ }^{(8,19)}$, contudo os estrógenos parecem apresentar pouca influência no desenvolvimento dos leiomiomas bucais, visto que não se observa predominância dessas lesões em mulheres ${ }^{(8,22,38)}$. Entretanto, por se tratar de lesão rara, a distribuição quanto ao sexo poderia ser modificada em decorrência do estudo de novos casos. Os leiomiomas apresentados neste trabalho acometeram mulheres.

A natureza neoplásica dos leiomiomas ${ }^{(13,19,34)}$ é questionada por alguns autores que discutem a possibilidade de o leiomioma vascular bucal representar uma fase final do processo progressivo de maturação iniciado no hemangio$\mathrm{ma}^{(12)}$, sendo, portanto, uma lesão hamartomatosa(12, 21).

Há divergências na literatura quanto ao sítio de predileção da neoplasia bucal. Alguns autores relatam que o leiomioma vascular é mais comumente localizado na língua e, em seguida, palato, mucosa da bochecha e lábio inferior ${ }^{(8,}$ ${ }^{14,43)}$. Entretanto, Savage et al.(37) revisaram a literatura e afirmaram que os sítios de maior acometimento são lábios, palato, língua e mucosa da bochecha. Por outro lado, Baden et al. ${ }^{(2)}$ encontraram maior ocorrência em lábios, seguidos de língua, bochecha e palato. Os dois casos relatados neste estudo apresentaram-se como leiomioma vascular em ventre lingual.

Assim, considerando a prevalência quanto ao gênero, os leiomiomas vasculares da cavidade bucal apresentam

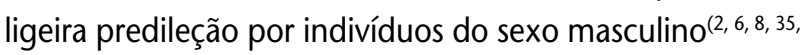
37). Entretanto, quando localizados em outras regiões, são mais freqüentes em mulheres ${ }^{(13,17)}$.

Quanto à faixa etária, embora possam ocorrer em qualquer idade ${ }^{(6,8,43)}$, são mais freqüentes na quinta e na sexta décadas de vida ${ }^{(6,35)}$, com média de 45 anos de idade ${ }^{(6)}$. As pacientes do estudo encontravam-se nessa faixa etária, o que corresponde aos dados apresentados na literatura.

A maioria dos casos de leiomioma bucal apresenta-se clinicamente como nódulos assintomáticos ${ }^{(1,6,7,23,43)}$, uma característica também observada nos casos deste estudo. Outras características clínicas são aspecto nodular delimitado, superficial, com pequeno diâmetro, e crescimento lento ${ }^{(21)}$. Essas características foram coincidentes com as dos casos descritos, exceto quanto à localização em profundidade das lesões, que se apresentavam como nódulos submucosos.

Quando massas teciduais benignas na língua são consideradas, diversas lesões devem ser incluídas no diagnóstico diferencial, como os linfangiomas e os hemangiomas, que podem ser localizados profundamente, embora mais fre- 
qüentemente se apresentem como lesões superficiais e com coloração bem característica. Por fim, os lipomas e as lesões reacionais também podem ser observados nessa região, embora essas últimas tenham aspectos inflamatórios, muitas vezes associados com sintomatologia dolorosa ${ }^{(33)}$.

Para o diagnóstico diferencial clínico, algumas neoplasias de tecido conjuntivo devem ser consideradas, incluindo neurilemoma, neuroma de amputação, lesões de glândulas salivares, lipoma e fibroma, entre outras ${ }^{(43)}$.

O tratamento de escolha para essas lesões é a remoção completa $^{(2,6,23,44)}$, o que resulta em poucas recorrências ${ }^{(8)}$ e prognóstico excelente ${ }^{(2)}$. Na literatura há o relato de uma recorrência duas semanas após a cirurgia ${ }^{(40)}$, provavelmente devido à excisão incompleta da neoplasia ${ }^{(27)}$.

Os aspectos histopatológicos observados nos casos relatados incluíram numerosos vasos de paredes espessadas e feixes de células fusiformes, com núcleos alongados e, por vezes, de extremidades rombas, características similares às descritas nos trabalhos consultados ${ }^{(6,27,34)}$.

A distinção entre leiomioma e leiomiossarcoma de baixo grau de malignidade pode ser complicada. A diferenciação morfológica baseia-se no grau de pleomorfismo celular, necrose, hipercromatismo, atipia nuclear e, principalmente, na presença de mitoses ${ }^{(18,36,39)}$. Como parâmetro, cinco ou mais mitoses em campo de maior aumento devem ser observadas ${ }^{(34)}$. O primeiro caso, embora tenha apresentado dissociação de fibras musculares esqueléticas na periferia, exibiu raras mitoses e aspectos histológicos condizentes com benignidade.

As lesões de células fusiformes apresentam ampla variação morfológica, explicada pela sua histogênese a partir de células mesenquimais indiferenciadas durante a embriogênese. Como tanto os fibroblastos quanto os leiomiócitos são células fusiformes, a distinção entre estas células pode ser difícil(2).

Neoplasias de origem neural, como neurilemoma, neurofibroma e tumor de células granulares, lesões usualmente circunscritas e com relatos de ocorrência na língua, devem ser pesquisadas e incluídas no diagnóstico diferencial devido aos seus aspectos histopatológicos $2(2,6,9,33)$.

Neste estudo utilizou-se a coloração tricrômica de Masson no segundo caso, embora, segundo Baden et al.(2), essa coloração não seja confiável, pois depende de fixação adequada e da técnica utilizada, podendo fornecer resultados falso-positivos. Por sua vez, a análise imuno-histoquímica apresenta maior especificidade quando os marcadores são selecionados adequadamente e utilizados sob condições controladas e padronizadas $(2,27,41)$.
Na literatura há relatos de casos de leiomioma vascular bucal diagnosticados com base nos aspectos morfológi$\cos ^{(20,21,24)}$. Com a imuno-histoquímica, novos estudos associam essa técnica aos achados microscópicos, resultando em diagnósticos precisos e confiáveis que até alteram diagnósticos prévios.

Baden et al.(2), ao realizarem reações imuno-histoquímicas em seis casos de leiomioma, verificaram que havia entre eles casos de neurofibroma, miofibroma e tumor de células granulares.

Há ainda o emprego de técnicas de biologia molecular que permitem o estudo genético dessas lesões, objetivando aprofundar os conhecimentos sobre sua etiopatogênese ${ }^{(31)}$.

Dessa forma, a técnica imuno-histoquímica tornouse importante ferramenta para o diagnóstico, podendo ser utilizada na identificação do leiomioma. Para tanto, podem-se empregar anticorpos contra actina de músculo liso, desmina e vimentina(2).

No caso 2 as células neoplásicas foram positivas para vimentina, resultados também encontrados na literatura ${ }^{(2,}$ 28). Esse marcador de células de origem mesenquimal tem sido reconhecido nessas lesões, embora não seja específico para células musculares lisas(2). Em adição, as células foram negativas para AE1/AE3, marcadores de citoqueratina ${ }^{(15)}$, evidenciando, juntamente com a positividade para vimentina, que a lesão não possui natureza epitelial, mas sim mesenquimal. Dessa maneira, foram excluídas as neoplasias de origem epitelial. Já no caso 1, os aspectos morfológicos por si só excluíam a possibilidade de origem epitelial. Portanto, o emprego desses marcadores não foi necessário.

Neste estudo também foi utilizado o anticorpo antidesmina no caso 1, cuja expressão foi negativa. A desmina é um filamento intermediário que caracteriza células musculares lisas maduras, miocárdio e células musculares esqueléticas, sendo utilizada, portanto, na identificação de neoplasias de origem muscular ${ }^{(3)}$. Entretanto, na literatura, foram encontrados resultados variados para marcação da desmina, que podem ou não estar presentes nos casos de leiomioma ${ }^{2,28,}$ 34). Essa variação, provavelmente, reflete graus diferentes de maturação e diferenciação celulares.

A marcação para proteína S-100 foi negativa nos dois casos estudados. Apesar de inicialmente se acreditar que a proteína S-100 estava presente apenas em tecidos neurais, sabe-se que tal proteína não é específica, pois é encontrada também em outras células não-neurais, como as mioepiteliais e as ductais de glândulas mamárias e sudoríparas, 
além de glândulas salivares ${ }^{(30)}$. A negatividade da reação para essa proteína é um indício para a possível exclusão do diagnóstico de neurofibroma.

As células neoplásicas das duas lesões relatadas foram negativas para CD-34, que marcou apenas células endoteliais do estroma, demonstrando que a proliferação celular não era originada dessas células.

No caso 1 empregou-se também o marcador de células endoteliais CD-31, que apresentou imunoexpressão semeIhante. A negatividade da reação para CD-34 descartou a hipótese de tumor fibroso solitário.

As duas neoplasias estudadas foram positivas para actina de músculo liso. Logo as hipóteses de neurofibroma e/ou tumor fibroso solitário foram descartadas.
Devido à expressão positiva para actina de músculo liso, concluiu-se que as células da neoplasia originavam-se de músculo liso, provavelmente da parede dos vasos sangüíneos, nos dois casos relatados.

\section{Conclusão}

A descrição e o estudo dos perfis histopatológico e imuno-histoquímico do leiomioma vascular bucal são relevantes em razão de sua raridade e similaridade com outras lesões de maior ocorrência na cavidade bucal.

A análise imuno-histoquímica é uma ferramenta importante para o diagnóstico final do leiomioma, e os anticorpos empregados neste estudo foram eficientes na distinção entre essa lesão e outros tumores de células fusiformes.

\section{Referências}

I. ANASTASSOV, G.E.; DAMME, P. A. Angioleiomyoma of the upper lip: report of a case. Int J Oral Maxillofac Surg, v. 24, n. 4, p. 30I-2, 1995.

2. BADEN, E.; DOYLE, J.L.; LEDERMAN, D.A. Leiomyoma of the oral cavity: a light microscopic and immunohistochemical study with review of the literature from 1884 to 1992. Eur J Cancer B Oral Oncol, v. 30, n. I, p. I-7, 1994.

3. BARWICK, K.W. Other intermediate filaments. In: TRUE, L.D. Atlas of diagnostic immunohistopathology. New York: J.B. Lippicott Company, 1990. p. 4.I-4.16.

4. BERTOLINI, F. et al. Mandibular intraosseous leiomyoma in a child: report of a case. J Clin Pediatr Dent, v. 27, n. 4, p. 385-7, 2003

5. BRIZUELA, A.F.; LEWIN, L.; FERRARIO, F. Leiomioma bilateral en paladar duro. La tribuna odontológica, v. 56, p. 274-6, 1972.

6. BROOKS, J.K. et al. Clinicopathologic characterization of oral angioleiomyoma. Oral Surg Oral Med Oral Pathol Oral Radiol Endod, v. 94, n. 2, p. 221-7, 2002.

7. BURKES, E.J. Vascular leiomyoma of the mandible: report of a case. J Oral Maxillofac Surg, v. 53, n. I, p. 65-6, 1995.

8. CHERRICK, H.M.; DUNLAP, C.L.; KING, O.H. Leiomyomas of the oral cavity. Oral Surg, v. 35, n. I, p. 54-66, 1973.

9. DAMM, D.D., NEVILLE, B.W. Oral leiomyomas. Oral Surg Oral Med Oral Pathol, v. 47, n. 4, p. 343-7, 1979.

10. DAS GUPTA, T.K.; CHAUDHURI, P.K. Tumors of the muscle tissue. In: Tumors of the soft tissues. Stamford, Connecticut: Appleton \& Lange, 1998. p. 309-42.

I I. DAVIS, G.B. Angiomyoma of the palate. Int J Oral Surg, v. $9, \mathrm{n}$. 6, p. 484-5, 1980.

12. DUIG, J.T.; AYER, J.P.Vascular leiomyoma: a study of 61 cases. Arch Pathol, v. 68, p. 424-30, 1959.

13. ENZINGER, F.M; WEISS, S.W. Angiomyoma. In: Soft tissue tumours. Mosby, 1995. p. 469-70.
14. EPIVATIANOS, A.; TRIGONIDIS, G.; PAPANAYOTOU, P. Vascular leiomyoma of the oral cavity. J Oral Maxillofac Surg, v. 43, n. 5, p. 377-82, 1985.

15. ERLANDERSON, R.A. Diagnostic immunohistochemistry of human tumors. Am J Surg Pathol, v. 8, n. 8, p. 615-24, 1984.

16. ESGUEP, A.; SOLAR, M. Oral vascular leiomyoma: report of 5 cases and review of the literature. J Oral Medicine, v. 4I, n. 2, p. 126-9, 1986.

17. FARMAN, A.G. Benign smooth muscle tumours. S Afr Med J, V. 49, n. 33, p. 1333-40. 1975.

I8. FLETCHER, C.D., KILPATRICK, S.E., MENTZEL,T.The difficulty in predicting behavior of smooth-muscle tumors in deep soft tissue. Am J Surg Pathol, v. 19, n. I, p. I 16-7, 1995.

19. FOX, H.; BURCKLY, H. The female genital tract and ovaries. In: McGEE, J.O.; ISAACSON, P.G.; WRIGHT, N.A. Oxford Textbook of pathology: pathology of systems. New York: Oxford University Press, 1992, p. I565-639.

20. GILES, A.D.; GOSNEY, M.B.E. Oral angiomyoma: a case report. Br J Oral Surg, v. 20, n. 2, p. 142-6, 1982.

21. GUTMANN, J. et al. Angiomyoma of the oral cavity. Oral Surg Oral Med Oral Pathol, v. 38, n. 2, p. 269-73. 1974.

22. HACHISUGAT.; HASHIMOTO H.; ENJOII M. Angioleiomyoma: a clinicopathologic reappraisal of 562 cases. Cancer, v. 54, n. I, p. 126-30, 1984.

23. INABA, H. et al. Painless mass of the cheek. Oral Surg Oral Med Oral Pathol Oral Radiol Endod, v. 95, n. I, p. 3-6, 2003.

24. KELLY, D.E.; HARRIGAN, W.F. Leiomyoma of the tongue: report of case.J Oral Surg, v. 35, n. 4, p. 316-8, 1977.

25. LEUNG, K.; WONG, D.Y.; LI, W. Oral leiomyoma: case report. J Oral Maxillofac Surg, v. 48, n. 7, p. 735-8, 1990.

26. LIANG, H. et al. Intraosseous oral leiomyoma: systematic review and report of one case. Dentomaxillofac Radiol, v. 32, n. 5 , p. 285-90, 2003. 
27. LLORIA-BENET, M. et al. Oral leiomyoma: a case report. Med Oral, v. 8, n. 3, p. 2I5-9, 2003.

28. MAEDA, Y. et al. Angiomyoma of the upper lip: report of a case with electron microscopic and immunohistochemical observation. Br J Oral Med Surg, v. 27, n. 3, p. 236-42, 1989.

29. MCGOWAN, D.A.; JONES, J.H.Angioma (vascular leiomyoma) of the oral cavity. Oral Surg Oral Med Oral Pathol, v. 27, n. 5, p. 649-52, 1969.

30. NAKAJIMA, T. et al. An immunoperoxidase study of S-100 protein distribution in normal and neoplastic tissues. Am J Surg Pathol, v. 6, n. 8, p. 7I5-27, 1982.

3।. NASCIMENTO, E.N. et al. Protocolo da extração de DNA de material parafinado para análise de microssatélites em leiomioma.J Bras Patol Med Lab, v. 39, n. 3, p. 253-5, 2003.

32. NATIELLA, J.R.; NEIDERS, M.E.; GREENE, G.W. Oral leiomyoma: report of six cases and a review of the literature. J Oral Patho, v. I I, n. 5, p. 353-65, 1982.

33. NELSON, W.; CHUPREVICH, T;; GALBRAITH, D.A. Enlarging tongue mass.J Oral Maxillofac Surg, v. 56, n. 2, p. 224-7, 1998.

34. NEVILLE, B.W. et al. Oral and maxillofacial pathology. 2. ed. Philadelphia: Saunders Company, 2002.

35. REICHART, P.; REZNIK-SCHULLER, H. The ultrastructure of an oral angiomyoma.J Oral Pathol, v. 6, n. I, p. 25-34, 1977.
36. REIS, R.S.A. et al. Leiomyoma of the tongue: case report. RPG Rev Pos-Grad, v. 9, n. 2, p. I 86-90, 2002

37. SAVAGE, N.W. et al. Oral vascular leiomyoma: review of the literature and report of two cases. Austr Dent Journal, v. 28, n. 6, p. 346-5I, 1983.

38. SHETTY, S.C. et al. Angioleiomyoma in the tonsil: an uncommon tumour in a rare site. Br J Oral Maxillofac Surg, v. 40, n. 2 . p. |69-7|, 2002

39. SOBRAL, A.P.V. et al.Leiomiossarcoma de boca: estudo histoquímico e imuno-histoquímico de dois casos clínicos. J Bras Patol Med Lab, v. 40, n. 5, p. 358-63, 2004.

40. SVANE,T.J. et al. Oral leiomyomas: review of the literature and report of a case of palatal angioleiomyoma.J Periodontol, v. 57, n. 7, p. 433-5. 1986.

4I. TOIDA, M.; KOIZUMI, H.; SHIMOKAWA, K. Painful angiomyoma of oral cavity: report of a case and review of the literature.J Oral Maxillofac Surg, v. 58, p. 450-3, 2000.

42. WEISS, S.W. Histological typing of soft tissue tumours. London: WHO Springer-Verlag, 1994

43. WERTHEIMER-HATCH, L. et al. Tumors of the oral cavity and pharynx. World J Surg, v. 24, p. 395-400, 2000.

44.WHITE, D.K. et al. Primary angioleiomyoma of the mandibuble. J Oral Maxillofac Surg, v. 43, p. 640-4, 1985.

\begin{tabular}{l|l} 
Endereço para correspondência \\
\hline Vanessa Ávila Sarmento Silveira \\
Departamento de Biociências e Diagnóstico Bucal da Fa- \\
culdade de Odontologia da Universidade Estadual Paulista \\
(UNESP) - Campus de São José dos Campos \\
Rua Engenheiro Francisco José Longo, 777 \\
CEP 12245-000 - São José dos Campos-SP \\
e-mail: vassilveira@yahoo.com.br
\end{tabular}

